\title{
Brodmann's Area
}

National Cancer Institute

\section{Source}

National Cancer Institute. Brodmann's Area. NCI Thesaurus. Code C94868.

One of 47 numbered regions within a map of the cerebral cortex. Each is characterized by a particular cell organization and also corresponds to a particular cortical function. 